\title{
Oxaliplatin-rapamycin combination was superior to mono-drug in treatment of hepatocellular carcinoma both in vitro and in vivo
}

\author{
X. Z. LI ${ }^{1}$, Y. L. SUN ${ }^{2, *}$, L. Q. CAO ${ }^{3}$, M. J. LI ${ }^{1, *}$ \\ ${ }^{1}$ Department of Radiotherapy, Liaocheng People's Hospital, Liaocheng, Shandong, China; ${ }^{2}$ Department of Medical Oncology, Shandong Cancer \\ Hospital and Institute, Jinan, Shandong, China; ${ }^{3}$ Department of Pathology, Dezhou People's Hospital, Dezhou, Shandong, China.
}

*Correspondence: sunyulansd@hotmail.com,lclimj@outlook.com

Received February 13, 2016 / Accepted July 18, 2016

\begin{abstract}
The presented study aimed to investigate the antitumor efficacy of combination of oxaliplatin with rapamycin, an mTOR inhibitor, in hepatocellular carcinoma (HCC). The activation status of mTOR pathway was first examined in HCC cell lines HepG2, BEL7402, and HuH7 using Western blotting. Effects of rapamycin, oxaliplatin, and their combination on the proliferation of HCC cells were determined in vitro using MTT assay and in vivo using a nude mice model bearing HepG2 xenografts. Drug-induced cell apoptosis was examined by flow cytometry. Expression of apoptosis-related protein was determined by Western blotting. We observed that mTOR pathway was activated in all three cell lines used in the current study. MTT assay demonstrated that oxaliplatin in combination with rapamycin synergistically inhibited the proliferation of HCC cells. The combination regimen reduced terminal tumor burden more efficiently than the corresponding monotherapy. The percentages of apoptotic cells and the expression levels of apoptosis-related proteins including cleaved caspase-9, -3, and PARP were significantly higher in combination-treatment groups than those in mono-drug-treatment groups. The ratios of Bax/ Bcl-2 in cells exposed to both oxaliplatin and rapamycin were significantly increased compared to those in cells subjected to oxaliplatin or rapamycin alone treatment. Results obtained in the presented study suggested that combination of oxaliplatin and rapamycin was superior to mono-drug and may have a potential value in treatment of HCC.
\end{abstract}

Key words: HCC, apoptosis, mTOR, chemotherapy

Hepatocellular carcinoma (HCC) is the sixth most common cancer in the world and the third leading cause of cancer-related death. Curative treatments including liver transplantation and hepatic resection are suitable only for fewer than $20 \%$ of HCC patients because most cases have progressed to an advanced stage with intra- or extra-hepatic metastasis when the disease is diagnosed $[1,2]$. As a palliative treatment, chemotherapy is an important strategy for patients with unresectable and metastatic HCC. Currently, sorafenib is the only systemic therapy which demonstrates survival benefit in the context of randomized controlled trials [3]. However, its efficacy remains moderate and only about $45 \%$ patients' condition could be controlled in a short term [3]. Alternative chemotherapeutic regimens are highly needed for those patients who are tolerant or fail to sorafenib treatment.

Oxaliplatin is a third-generation platinum compound with lower toxicity compared to cisplatin and carboplatin [4]. Oxaliplatin-based chemotherapy for advanced HCC has at- tracted a great attention worldwide in recent years. Oxaliplatin as a single agent was well tolerated with acceptable toxicity in patients with advanced HCC in a phase II clinical study. In addition, the treatment achieved 1 partial response (PR) and 16 stable disease (SD) in 36 tested patients and median survival of 6 months, suggesting that oxaliplatin could be an active and promising agent for HCC treatment [5]. Enhancement of the efficacy of oxaliplatin by its use in combination regimens is a rational strategy for prolonging the survival of patients. In fact, a number of different oxaliplatin-based regimens have been investigated in advanced HCC patients, such as oxaliplatin/gemcitabine (GEMOX) and oxaliplatin/ fluoropyrimidines (FOLFOX4) chemotherapies. However, these combined regimens achieved a small range of tumor responses [6, 7]. Further investigation of oxaliplatin in combination with other agents is warranted.

Rapamycin is an mTOR inhibitor that shows potent antitumor activity in a variety of malignances including HCC 
in preclinical and clinical studies [8-11]. Notably, rapamycin and its derivatives have been demonstrated to enhance the efficacy of cytotoxic chemotherapeutic drugs including oxaliplatin, topotecan, and 5-fluorouracil (5-FU) in the treatment of various types of cancers such as gastric and colon cancers $[8,12-15]$. Given that mTOR signal pathway was shown to be hyperactivated in HCC cell lines [16, 17], we investigated the efficacy of combination of rapamycin and oxaliplatin against HCC in the present study.

\section{Materials and methods}

Chemicals and agents. Rapamycin and oxaliplatin were purchased from Sigma-Aldrich (St. Louis, MO, USA). Rapamycin oral solution (Rapamune) and Oxaliplatin for injection (Eloxatin') were obtained from Wyeth Pharmaceuticals Inc. (Rouses Point, NY, USA) and Sanofi-Aventis France (Paris, France), respectively. For in vitro assay, rapamycin and oxaliplatin were firstly dissolved in dimethyl sulfoxide (DMSO; Sigma-Aldrich) and further diluted in phosphate buffer solution (PBS) before use. For in vivo study, rapamycin oral solution and oxaliplatin for injection were given by gavage and intravenous injection, respectively.

Cell line and cell culture. HCC cell lines HepG2, BEL7402, and $\mathrm{HuH7}$ was purchased from China Cell Bank, Shanghai, China. The cells were maintained in Iscove's modified Dulbecco's medium (IMDM; Thermo Fisher Scientific, Waltham, MA, USA) supplemented with $10 \%$ fetal bovine serum (FBS; Evergreen Biotechnology, Hangzhou, Zhejiang, China) at $37^{\circ} \mathrm{C}$ in a humid atmosphere (5\% CO2-95\% air). Cells were harvested by brief incubation in $0.02 \%(\mathrm{w} / \mathrm{v})$ ethylenediaminetetraacetic acid (EDTA) in phosphate buffered saline (PBS).

Cell proliferation assay. Cells $\left(1 \times 10^{4}\right.$ per well $)$ seeded in 96-well plates were exposed to rapamycin, oxaliplatin, or their combination for $72 \mathrm{~h}$. Then the medium was removed and the wells were washed with PBS. The 3-(4,5-dimethylthiazol-2-yl)2,5-diphenyltetrazolium bromide (MTT) assay was performed by adding $20 \mu \mathrm{L}$ of MTT ( $5 \mathrm{mg} / \mathrm{mL}$, Sigma, USA) for $4 \mathrm{~h}$. Light absorbance of the solution was measured at $570 \mathrm{~nm}$ on a microplate reader (Perkin-Elmer, USA).

Annexin V/FITC/PI staining assay. The apoptotic cells were estimated by determining the levels of phosphatidylserine on cell surface. Cells seeded in $25-\mathrm{cm}^{2}$ culture flasks ( 1.5 $\times 10^{5}$ cells per $\mathrm{mL} ; 6 \mathrm{~mL}$ per flask) were exposed to vehicle, rapamycin alone $(5,10$, and $20 \mathrm{nM}$, respectively), oxaliplatin alone $(2.5,5$, and $10 \mu \mathrm{M}$, respectively), or their combinations for $48 \mathrm{~h}$. The levels of phosphatidylserine were determined by using Annexin-V/FITC and PI kit (Labtek, Dalian, Liaoning, China). The experiment was performed on a FACScan flow cytometry. The population of apoptotic cells was estimated by comparing to the vehicle control.

Western blotting. Cells $\left(3.0 \times 10^{5}\right.$ per well $)$ seeded in 6-well plates were exposed to vehicle, rapamycin alone (10 $\mathrm{nM})$, oxaliplatin alone $(5 \mu \mathrm{M})$, or their combination for 48 h. Cells were harvested and cell lysates $(30 \mu \mathrm{g}$ of protein per lane) were fractionated by $10 \%$ SDS-PAGE. The proteins were electro-transferred onto nitrocellulose membrane and the protein levels were detected using the primary antibodies against phosphorylated mTOR, p70S6K, 4E-BP1, caspase-3 and -9, cleaved PARP, Bcl-2, Bax, and $\beta$-actin (Santa Cruz, Dallas, TX, USA) with appropriate dilution. The bound antibodies were visualized using an enhanced chemiluminescence reagent and quantified by densitometry using ChemiDoc XRS+ image analyzer (Bio-Rad, Hercules, CA, USA). Densitometric analyses of bands were adjusted with $\beta$-actin as loading control. The percentages of increase or decrease of protein were estimated by comparison to the vehicle control (100\%).

In vivo inhibition of tumor growth. The in vivo efficacy of combination of rapamycin and oxaliplatin was assessed in a HepG2 xenograft mouse model. Balb/c athymic (nu+/ $\mathrm{nu}+$ ) female mice, 4-6 weeks of age, were purchased from the Animal Center of China Academy of Medical Sciences (Beijing, China). The animals were housed under pathogenfree conditions. The research protocol was in accordance with the institutional guidelines of the Animal Care and Use Committee.

Tumors were generated by harvesting HepG2 cells from mid-log phase cultures. Cells were then pelleted and resuspended in PBS to a final cell count of $5 \times 10^{7} / \mathrm{mL}$. A volume of $0.2 \mathrm{~mL}$ of the cell suspension was injected s.c. in the right flank of each mouse. After 7 days, when the tumor volume had reached approximately $0.1 \mathrm{~cm}^{3}$, all the mice were divided into 4 groups $(n=6)$ and then subjected to treatment. Group 1: mice were injected with $0.2 \mathrm{~mL}$ normal saline via tail vein. Group 2: rapamycin was administered p.o. at $1.5 \mathrm{mg} / \mathrm{kg}$. Group 3: oxaliplatin was administered i.v. at $10 \mathrm{mg} / \mathrm{kg}$. Group 4: mice were received $1.5 \mathrm{mg} / \mathrm{kg}$ of rapamycin (p.o.) and $10 \mathrm{mg} /$ $\mathrm{kg}$ of oxaliplatin (i.v.). Administration of vehicle, rapamycin, oxaliplatin, or their combination was performed daily for 21 days. Tumors were harvested at the end of experiment and tumor growth inhibition rates were defined as a percentage of the control tumor weight.

Statistical analysis. Data was expressed as mean \pm S.D. for three different determinations. Statistical significance was analyzed by one-way analysis of variance (ANOVA) followed by Dunnett's multiple range tests. $p<0.05$ was considered as statistically significant. Statistical analysis was performed using the SPSS/Win 12.0 software (SPSS, Inc, Chicago, IL, USA).

\section{Results}

Rapamycin-oxaliplatin combination enhanced antiproliferative effect. Given that mTOR signal transduction pathway has an essential role in the regulation of cell survival and suppression of this pathway may sensitize cancer cells to cytotoxic drugs [18], in the present study we first checked the expression of mTOR and its downstream molecules p70S6K and 4E-BP1 in HepG2, BEL7402, and HuH7 cells that were used in our study. As was expected, results of Western blotting showed that all these three cell lines express high levels of 
phosphorylated mTOR, p70S6K, and 4E-BP1, indicating that mTOR pathway is activated in the cells (data not shown).

Next we determined the anti-proliferation effect of rapamycin, oxaliplatin, and their combination in HepG2, BEL7402, and $\mathrm{HuH7}$ cells, respectively, using MTT assay. Cells were treated with increasing concentrations of rapamycin (1.25, $2.5,5.0,10$, and $20 \mathrm{nM})$, oxaliplatin $(0.625,1.25,2.5,5.0$, and $10 \mu \mathrm{M}$ ), or the combination of two drugs for $72 \mathrm{~h}$. The drug concentrations were determined based on the following consideration. The toxicity of rapamycin was dose-dependent and the maximum plasma concentrations should be maintained at a range of approximate $10-20 \mathrm{nM}$ in patients receiving organ transplantation $[19,20]$. Regarding oxaliplatin, the peak plasma concentration was approximate $10 \mu \mathrm{M}$ after administration of the recommendation dose of $130 \mathrm{mg} / \mathrm{m}^{2}$ [21]. Thus, concentrations of either rapamycin or oxaliplatin used in our study represent a range that typically bracket therapeutic dose levels achieved in the circulation. Results demonstrated that both rapamycin and oxaliplatin dose-dependently inhibited the proliferation of HCC cells. The maximum inhibition rates were determined to be $13.2 \%, 15.8 \%$, and $17.9 \%$ for rapamycin at $20 \mathrm{nM}$, and $55.4 \%, 58.3 \%$, and $62.5 \%$ for oxaliplatin at

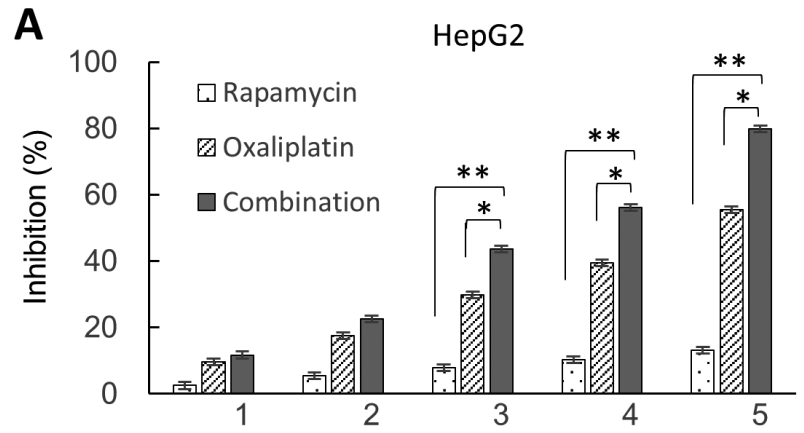

D
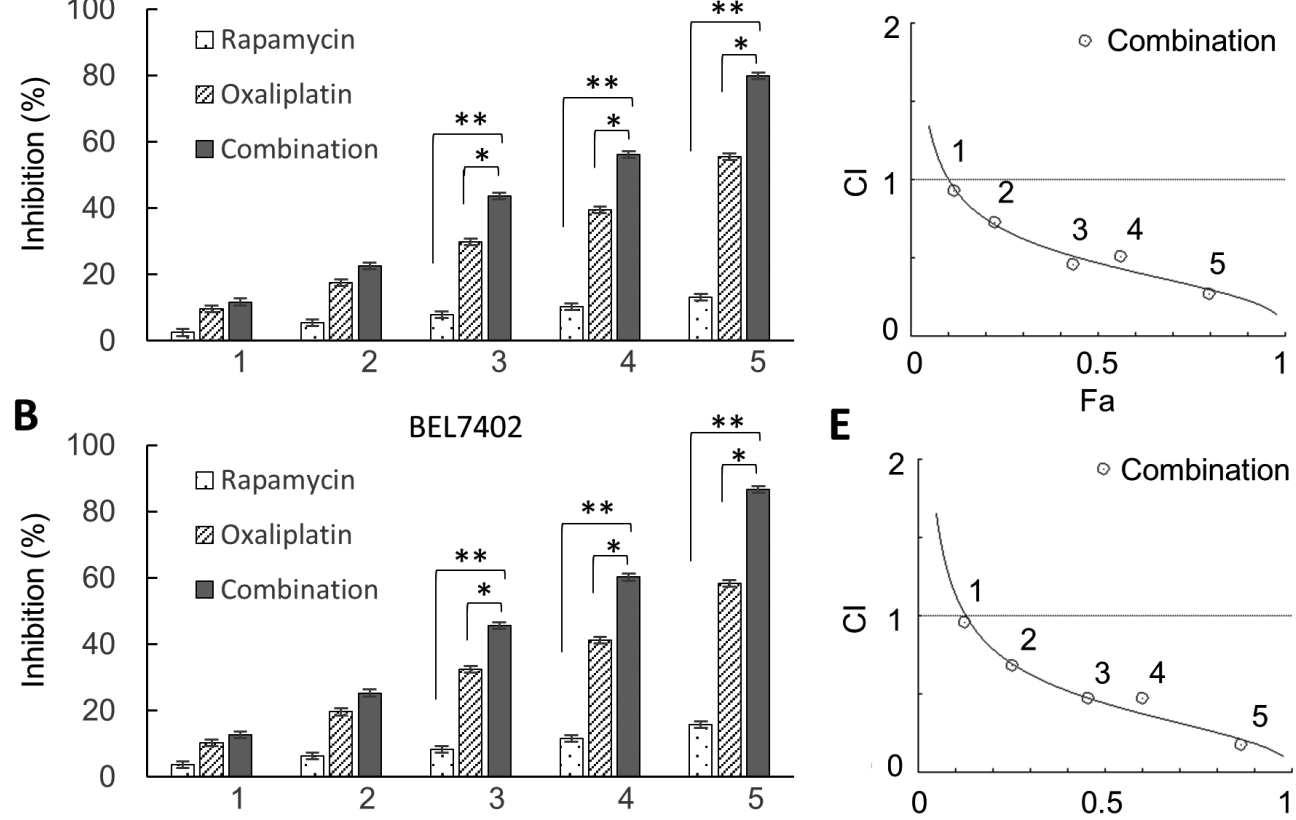

E

$\mathrm{Fa}$
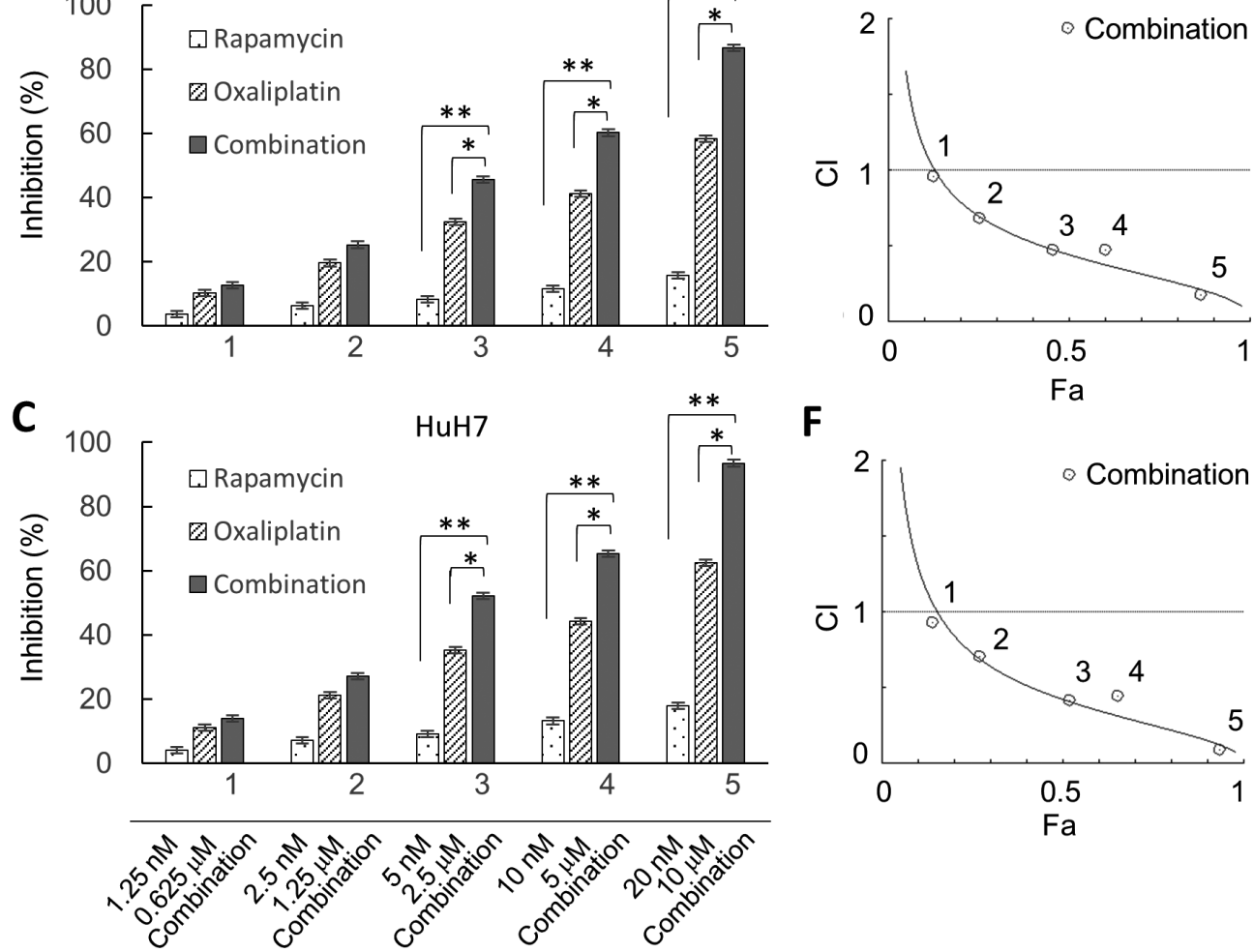

Figure 1. Effects of rapamycin, oxaliplatin, and their combination on the proliferation of HCC cells. The inhibition rates of rapamycin plus oxaliplatin were significantly higher than those of rapamycin or oxaliplatin at relatively high concentrations (oxaliplatin plus rapamycin: $2.5 \mu \mathrm{M}+5 \mathrm{nM}, 5.0 \mu \mathrm{M}+$ $10 \mathrm{nM}, 10 \mu \mathrm{M}+20 \mathrm{nM}$ ) in HepG2 cells (A), BEL7402 cells (B), and HuH7 cells (C). $p<0.05$, vs. oxaliplatin alone; $p<0.01$, vs. rapamycin alone. Combination Index Plot was generated by the CompuSyn software to determine drug interaction effects in HepG2 (D), BEL7402 (E), and HuH7 (F) cells. Combination index $(\mathrm{CI})$ values were all less than 0.7 at the above drug combinations. CI values between $0.3-0.7$ were regarded as 'synergism' and $<0.3$ as 'strong synergism'. Experiments were repeated for three times $(n=3)$. 
$10 \mu \mathrm{M}$, in HepG2, BEL7402, and HuH7, respectively (Figure 1). The inhibition rates of rapamycin plus oxaliplatin were significantly higher than that of rapamycin or oxaliplatin at relatively high concentrations (oxaliplatin plus rapamycin: $2.5 \mu \mathrm{M}+5 \mathrm{nM}, 5.0 \mu \mathrm{M}+10 \mathrm{nM}, 10 \mu \mathrm{M}+20 \mathrm{nM}$ ) (Figures $1 \mathrm{~A}$, $1 \mathrm{~B}$, and $1 \mathrm{C}$ ), and the maximum inhibition rates reached $79.9 \%$, 86.6\%, and 93.5\%, in HepG2, BEL7402, and HuH7 cells. We determined the level of p-p70S6K in cells treated with rapamycin alone or rapamycin plus oxaliplatin using Western blotting. Results demonstrated that rapamycin alone or combination with oxaliplatin dose-dependently reduced p-p70S6K level in HepG2, BEL7402, and HuH7 cells at concentrations of 5-20 nM, suggesting that mTOR signaling was suppressed at these concentrations (Supplementary Figure 1).

To explore whether the combined treatment had a synergistic impact on cell viability, the combination index (CI) values of each dose were calculated by the CompuSyn software. The results revealed that oxaliplatin exhibited an obvious synergistic effect in combination with rapamycin at relatively high concentrations (oxaliplatin plus rapamycin: $2.5 \mu \mathrm{M}+5 \mathrm{nM}$, $5.0 \mu \mathrm{M}+10 \mathrm{nM}, 10 \mu \mathrm{M}+20 \mathrm{nM}$ ) in HepG2 cells (Figure 1D). The synergistic effect between oxaliplatin and rapamycin were also observed in BEL7402 and $\mathrm{HuH7}$ cells, indicating that this was not a cell line-specific effect (Figures $1 \mathrm{E}$ and $1 \mathrm{~F}$ ). Taken together, our results suggest that oxaliplatin plus rapamycin synergistically increased anti-proliferative effect consistently in different HCC cell lines.

Rapamycin-oxaliplatin combination enhanced apoptotic cell death. Next we examined the effect of rapamycin plus oxaliplatin in soliciting cell apoptosis in HepG2, BEL7402, and HuH7 cells. Drug concentrations in combination groups were set at $5.0 \mathrm{nM}$ rapamycin plus $2.5 \mu \mathrm{M}$ oxaliplatin, $10 \mathrm{nM}$ rapamycin plus $5.0 \mu \mathrm{M}$ oxaliplatin, and $20 \mathrm{nM}$ rapamycin plus 10 $\mu \mathrm{M}$ oxaliplatin because these dose combinations showed synergistic antiproliferative effect in the MTT assay. Cells were treated with rapamycin alone $(5.0,10$, and $20 \mathrm{nM})$, oxaliplatin alone $(2.5,5.0$, and $10 \mu \mathrm{M})$, and the above drug combinations, respectively, for $48 \mathrm{~h}$ and then subjected to flow cytometry analysis. In HepG2 cells, the apoptotic cells in vehicle-treated group occupied $2.7 \%$ (Figure 2A). The percentages of apoptotic cells were increased by $1.5 \%, 2.7 \%$, and $4.1 \%$ after exposure to $5.0,10$, and $20 \mathrm{nM}$ rapamycin, respectively; by $5.9 \%, 9.5 \%$, and $15.2 \%$ after exposure to $2.5,5.0$, and $10 \mu \mathrm{M}$ oxaliplatin, respectively; by $11.2 \%, 15.6 \%$, and $25.9 \%$ after exposure to corresponding combination doses (Figure 2A and Supplementary Figure 2A). Statistical analysis showed that rates of apoptotic HepG2 cells in combination groups were significantly higher than those treated with rapamycin or oxaliplatin alone $(p<$ $0.01 v$ s. rapamycin, $p<0.05 v$ s. oxaliplatin) (Figure 2A). Similar results were obtained in other two cell lines. In BEL7402 cells, the apoptotic cells in vehicle-treated group occupied $2.3 \%$. The percentages of apoptotic cells were increased by $1.1 \%, 2.1 \%$, and $3.5 \%$ after exposure to $5.0,10$, and $20 \mathrm{nM}$ rapamycin, respectively; by $4.4 \%, 10.5 \%$, and $16.5 \%$ after exposure to 2.5 , 5.0 , and $10 \mu \mathrm{M}$ oxaliplatin, respectively; by $10.9 \%, 17.2 \%$, and
$27.7 \%$ after exposure to corresponding combination doses (Figure 2B and Supplementary Figure 2B). Statistical analysis showed that rates of apoptotic BEL7402 cells in combination groups were significantly higher than those treated with rapamycin or oxaliplatin alone $(p<0.01 v s$. rapamycin, $p<0.05$ vs. oxaliplatin) (Figure 2B). In HuH7 cells, the apoptotic cells in vehicle-treated group occupied $3.2 \%$. The percentages of apoptotic cells were increased by $2.3 \%, 3.5 \%$, and $4.6 \%$ after


Figure 2. Effects of rapamycin, oxaliplatin, and their combination on the apoptosis of HepG2 (A), BEL7402 (B), and HuH7 (C) cells. Cells were treated with vehicle, rapamycin alone $(5,10$, and $20 \mathrm{nM}$, respectively), oxaliplatin alone $(2.5,5$, and $10 \mu \mathrm{M}$, respectively), and their combinations for $48 \mathrm{~h}$ and then subjected to flow cytometry analysis. Percentages of apoptotic cells were significantly higher in combination-treatment groups than those in mono-drug-treatment groups. $p<0.05$, vs. oxaliplatin alone; $p<0.01, v s$. rapamycin alone. 
exposure to 5.0, 10, and $20 \mathrm{nM}$ rapamycin, respectively; by $8.6 \%, 12.5 \%$, and $18.7 \%$ after exposure to $2.5,5.0$, and $10 \mu \mathrm{M}$ oxaliplatin, respectively; by $12.9 \%, 18.6 \%$, and $27.3 \%$ after exposure to corresponding combination doses (Figure 2C and supplementary Figure 2C). Statistical analysis showed that rates of apoptotic HuH7 cells in combination groups were significantly higher than those treated with rapamycin or oxaliplatin alone ( $p<0.01 v s$. rapamycin, $p<0.05 v s$. oxaliplatin) (Figure 2C). These results suggested that rapamycin plus oxaliplatin obviously increased the rates of apoptotic HCC cells compared to mono-drug used alone.

Expression of apoptosis-related proteins in HCC cells. To confirm the effect of rapamycin plus oxaliplatin in soliciting cell apoptosis and investigate the underlying mechanisms, we examined the expression of apoptosisrelated proteins in HepG2 cells. Cells were incubated with rapamycin $(10 \mathrm{nM})$, oxaliplatin $(5 \mu \mathrm{M})$, or rapamycin $(10$ $\mathrm{nM})$ plus oxaliplatin $(5 \mu \mathrm{M})$ for $48 \mathrm{~h}$ and then subjected to Western blotting to analyze the expression of apoptosisrelated proteins including caspase 9 , caspase 3, PARP, Bcl-2, and Bax. Results demonstrated that when exposure to rapamycin, oxaliplatin, and their combination, the apoptotic proteins were significantly increased by $9.2 \%, 24.5 \%$, and $61.3 \%$, respectively, for cleaved caspase 9 (rapamycin, $p>$ 0.05 ; oxaliplatin, $p<0.05$; rapamycin plus oxaliplatin, $p<$ $0.01 v s$. the vehicle control); $6.5 \%, 28.8 \%$, and $68.9 \%$, respectively, for cleaved caspase 3 (rapamycin, $p>0.05$; oxaliplatin, $p<0.05$; rapamycin plus oxaliplatin, $p<0.01 v s$. the vehicle control); $3.9 \%, 32.6 \%$, and $135.9 \%$, respectively, for cleaved PARP (rapamycin, $p>0.05$; oxaliplatin, $p<0.05$; rapamycin plus oxaliplatin, $p<0.01 v s$. the vehicle control) (Figure 3). Statistical analysis showed that expression levels of cleaved caspase 9, caspase 3, and PARP in combination treatment group were significantly higher than those in rapamycin or oxaliplatin treatment group ( $p<0.01$, vs. rapamycin; $p<$ 0.05 vs. oxaliplatin) (Figure 3). Our results also showed that both rapamycin and oxaliplatin were capable of upregulating Bax and down-regulating Bcl-2 (Figure 3). Statistical analysis indicated that the ratio of $\mathrm{Bax} / \mathrm{Bcl}-2$ was increased by $25.9 \%, 54.2 \%$, and $77.8 \%$, respectively, when cells were exposed to rapamycin, oxaliplatin, and their combination. These results suggested that rapamycin-oxaliplatin combination enhanced cell apoptosis and this effect may be related with the increased ratio of $\mathrm{Bax} / \mathrm{Bcl}-2$.

Suppression of tumor growth in vivo. To assess the efficacy of rapamycin plus oxaliplatin regimen in vivo, a HepG2 human xenograft model was established and animals were randomly assigned to control group, rapamycin group, oxaliplatin group, and drug combination group. Rapamycin dosed orally at $1.5 \mathrm{mg} / \mathrm{kg}$ slightly inhibited tumor growth by $5.1 \%$ compared to control (Figure 4A). Oxaliplatin significantly reduced terminal tumor burden by $22.4 \%$ when dosed at 10 $\mathrm{mg} / \mathrm{kg}$ (Figure $4 \mathrm{~A}$ ). The rapamycin-oxaliplatin combination $(1.5$ and $10 \mathrm{mg} / \mathrm{kg}$, respectively) significantly reduced terminal tumor burden by $42.5 \%$ which is obviously higher than that of the corresponding monotherapy controls (Figure 4A). These data suggested that rapamycin and oxaliplatin dosed in combination acted efficiently in reducing overall tumor volume using the HepG2 xenograft model.

Oral rapamycin was generally well tolerated by mice with no significant loss of body weight ( $p>0.05 v s$. vehicle) (Figure 4B). Oxaliplatin injection resulted in a significant reduction in body weight ( $p<0.05 v s$. vehicle). A significant loss of body weight was also observed in mice receiving the rapamycin-oxaliplatin combination (Figure 4B). However, there was no significant difference in body weight between combined treatment group and oxaliplatin alone treatment group $(p>0.05)$.

\section{Control Rapamycin Oxaliplatin Combination}

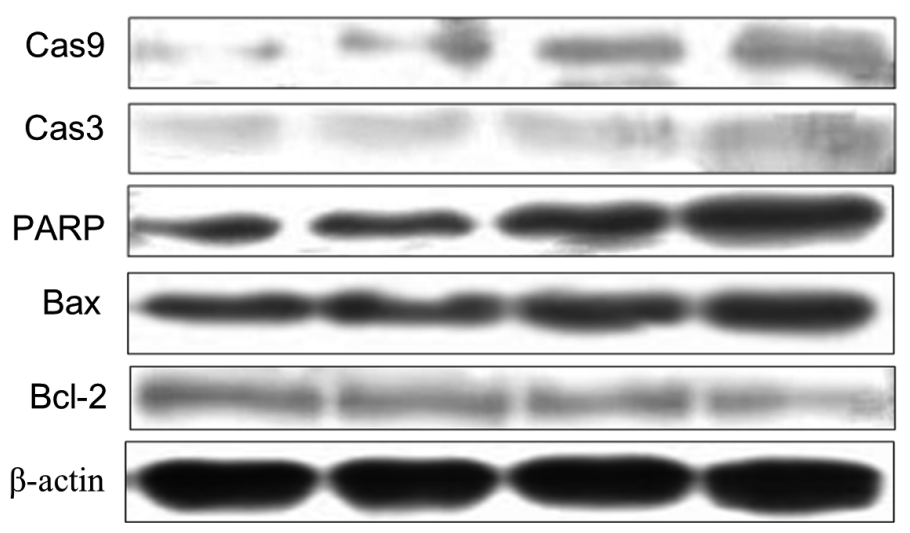



Figure 3. Effects of rapamycin, oxaliplatin, and their combination on apoptosis-related protein expression in HepG2 cells. Cells were treated with vehicle, rapamycin alone $(10 \mathrm{nM})$, oxaliplatin alone $(5 \mu \mathrm{M})$, and their combination for $48 \mathrm{~h}$ and then subjected to Western blotting assay. Left: immunostaining bands; Right: Quantitative comparison. Protein expression levels in control group were regarded as $100 \%$. 


\section{Discussion}

We investigated the antitumor effect of rapamycin plus oxaliplatin in HCC cells HepG2, BEL7402, and HuH7 cells in the present study. Cell proliferation assay showed that rapamycin-oxaliplatin combination had a synergistic effect in suppressing the proliferation of HCC cells. Flow cytometry assay demonstrated that this combination was more potent in soliciting cell apoptosis than rapamycin or oxaliplatin alone. The apoptosis-inducing effect was also evidenced by that combination treatment significantly increased the expression of cleaved caspase 9, caspase 3, and PARP. Further analysis showed that rapamycin-oxaliplatin combination treatment obviously increased the ratio of $\mathrm{Bax} / \mathrm{Bcl}-2$ compared to mono-drug used alone, which might account for its superior apoptosis-inducing effect. The antitumor efficacy of rapamycin plus oxaliplatin was finally confirmed in a HepG2 xenograft model. These results suggested that combination of rapamycin and oxaliplatin enhanced the antitumor efficacy compared to rapamycin or oxaliplatin alone in HCC cells HepG2, BEL7402, and $\mathrm{HuH7}$.

Oxaliplatin was approved for treatment of advanced HCC in China in 2013 on the ground of the results of EACH study in which oxaliplatin-based regimen (FOLFOX4) significantly improved the median progression-free survival (PFS) and OS compared with doxorubicin (PFS: 2.4 vs. 1.7 months; OS: 5.9 vs. 4.3 months) [6]. However, the response rate of FOLFOX4 regimen was only $8.6 \%$, indicating that quite a part of patients are not sensitive to this regimen [6]. Considering the heterogeneity of HCC, exploration of different oxaliplatin-based combined therapies will benefit more HCC patients. Rapamycin is a macrolide that produced by the bacteria Streptomyces hygroscopicus and is currently used to prevent rejection in organ transplantation in clinics including liver transplantation for HCC $[22,23]$. Studies in recent years demonstrated that it also has potential anti-HCC activity via inactivating the mammalian target of rapamycin (mTOR) [8, 9]. Results obtained in our study showed that addition of rapamycin to oxaliplatin significantly increased anti-HCC effects and did not give rise to obviously increased toxicity, which indicates that this combination may have a potential value in treatment of advanced HCC. Given the application of rapamycin in liver transplantation, its combination with oxaliplatin might also have the potential to treat recurrent HCC even after liver transplantation.

We observed that rapamycin-oxaliplatin combination significantly activated caspase 9 and caspase 3 in HepG 2 cells, suggesting this drug combination might induce cell apoptosis, at least in part, through the intrinsic mitochondria-mediated pathway. The crucial event for initiating this pathway is the distruction of mitochondrial membrane potential $\left(\Delta \Psi_{\mathrm{m}}\right)$ that is controlled by members of the Bcl-2 protein family and requires the activation of one of its pro-apoptotic members such as Bax [24]. The collapse of $\Delta \Psi_{\mathrm{m}}$ allows release of signaling molecules from the space between outer and inner mitochondrial mem-


Figure 4. Effects of rapamycin, oxaliplatin, and their combination on the growth of HepG2 xenografts. Nude mice bearing HepG2 xenografts were received rapamycin alone $(1.5 \mathrm{mg} / \mathrm{kg} / \mathrm{day})$, oxaliplatin alone $(10 \mathrm{mg} / \mathrm{kg} /$ day), and their combination, respectively, for 21 days. Mice were weighed and sacrificed at the end of the experiment. Tumors were removed and weighed. ${ }^{\star} p<0.05$, vs. vehicle; ${ }^{\star *} p<0.01, v s$. vehicle.

branes into cytosol, and triggers caspase activation and other apoptotic processes. Bax is constitutively located within the mitochondrial membrane, whereas the activation of Bax also involves its translocation from cytosol to outer mitochondrial membrane and, subsequently, its insertion into membrane. Binding of p53 to Bax in outer mitochondrial membrane was found to catalyze Bax activation and cytochrome $c$ release from mitochondria $[25,26]$. Anti-apoptotic members (e.g. Bcl-2, $\mathrm{Bcl}$-xL) antagonize $\Delta \Psi_{\mathrm{m}}$ collapse by maintaining membrane integrity. Pro-apoptotic members (e.g. Bax, Bak) are capable of inducing $\Delta \Psi_{\mathrm{m}}$ collapse. In these processes, the release of cytochrome $c$ is associated with the activation of caspase-9, caspase- 3 and cleavage of PARP and thereby triggers cancer cells to apoptosis. It was observed in the current study that both rapamycin and oxaliplatin were capable of upregulat- 
ing Bax expression and downregulating Bcl-2 expression, however, the ratio of $\mathrm{Bax} / \mathrm{Bcl}-2$ is obviously higher in the combined treatment group than that in rapamycin or oxaliplatin alone treatment group. Thus the induction of apoptosis by rapamycin-oxaliplatin combination is considered to activate the intrinsic mitochondria-mediated pathway through altering the expression levels of Bax and Bcl-2.

In conclusion, combination of rapamycin and oxaliplatin showed synergistic effect in suppressing cell proliferation and enhanced cell apoptosis in HCC cells, which might be related with activation of the intrinsic mitochondria-mediated pathway. Further studies are warranted to comprehensively evaluate the efficacy and toxicity of this regimen in the future.

Supplementary information is available in the online version of the paper.

\section{References}

[1] JOHNSON PJ. Non-surgical treatment of hepatocellular carcinoma. HPB (Oxford) 2005; 7: 50-55. http://dx.doi. org/10.1080/13651820410024076

[2] GAO J, FENG X, INAGAKI Y, SONG P, KOKUDO N et al. Des-gamma-carboxy prothrombin and c-Met were concurrently and extensively expressed in hepatocellular carcinoma and associated with tumor recurrence. Biosci Trends 2012; 6: 153-159. http://dx.doi.org/10.5582/bst.2012.v6.4.153

[3] LLOVET JM, RICCI S, MAZZAFERRO V, HILGARD P, GANE E et al. Sorafenib in advanced hepatocellular carcinoma. N Engl J Med 2008; 359: 378-390. http://dx.doi. org/10.1056/NEJMoa0708857

[4] RUGGIERO A, TROMBATORE G, TRIARICO S, ARENA R, FERRARA $P$ et al. Platinum compounds in children with cancer: toxicity and clinical management. Anticancer Drugs 2013; 24: 1007-1019. http://dx.doi.org/10.1097/ CAD.0b013e3283650bda

[5] YEN Y, LIM DW, CHUNG V, MORGAN RJ, LEONG LA et al. Phase II study of oxaliplatin in patients with unresectable, metastatic, or recurrent hepatocellular cancer: a California Cancer Consortium Trial. Am J Clin Oncol 2008; 31:317-322. http://dx.doi.org/10.1097/COC.0b013e318162f57d

[6] QIN S, CHENG Y, LIANG J, SHEN L, BAI Y et al. Efficacy and safety of the FOLFOX4 regimen versus doxorubicin in Chinese patients with advanced hepatocellular carcinoma: a subgroup analysis of the EACH study. Oncologist 2014; 19: 1169-1178. http://dx.doi.org/10.1634/theoncologist.2014-0190

[7] LIU Y, YUE H, XU S, WANG F, MA N et al. First-line gemcitabine and oxaliplatin (GEMOX) plus sorafenib, followed by sorafenib as maintenance therapy, for patients with advanced hepatocellular carcinoma: a preliminary study. Int J Clin Oncol 2015; 20: 952. http://dx.doi.org/10.1007/s10147-015-0796-5

[8] LU X, WEI H, ZHANG X, ZHENG W, CHANG C et al. Rapamycin synergizes with low-dose oxaliplatin in the HCT116 colon cancer cell line by inducing enhanced apoptosis. Oncol Lett 2011; 2: 643-647.
[9] KAWADA JI, ITO Y, IWATA S, SUZUKI M, KAWANO Y et al. mTOR Inhibitors Induce Cell-Cycle Arrest and Inhibit Tumor Growth in Epstein-Barr Virus-Associated T and Natural Killer Cell Lymphoma Cells. Clin Cancer Res 2014. 20: 5412-5422. http://dx.doi.org/10.1158/1078-0432.CCR-13-3172

[10] PENG DJ, WANG J, ZHOU JY, WU GS. Role of the Akt/mTOR survival pathway in cisplatin resistance in ovarian cancer cells. Biochem Biophys Res Commun 2010; 394: 600-605. http:// dx.doi.org/10.1016/j.bbrc.2010.03.029

[11] TOSO C, MERANI S, BIGAM DL, SHAPIRO AM, KNETEMAN NM. Sirolimus-based immunosuppression is associated with increased survival after liver transplantation for hepatocellular carcinoma. Hepatology 2010; 51: 1237-1243. http:// dx.doi.org/10.1002/hep. 23437

[12] RAJE N, KUMAR S, HIDESHIMA T, ISHITSUKA K, CHAUHAN D et al. Combination of the mTOR inhibitor rapamycin and CC-5013 has synergistic activity in multiple myeloma. Blood 2004; 104: 4188-4193. http://dx.doi.org/10.1182/blood2004-06-2281

[13] MATSUZAKI T, YASHIRO M, KAIZAKI R, YASUDA K, DOI Y et al. Synergistic antiproliferative effect of mTOR inhibitors in combination with 5-fluorouracil in scirrhous gastric cancer. Cancer Sci 2009; 100: 2402-2410. http://dx.doi. org/10.1111/j.1349-7006.2009.01315.x

[14] PENCREACH E, GUERIN E, NICOLET C, LELONG-REBEL I, VOEGELI AC et al. Marked activity of irinotecan and rapamycin combination toward colon cancer cells in vivo and in vitro is mediated through cooperative modulation of the mammalian target of rapamycin/hypoxia-inducible factor-1alpha axis. Clin Cancer Res 2009; 15: 1297-1307. http://dx.doi. org/10.1158/1078-0432.CCR-08-0889

[15] ACEVEDO-GADEA C, SANTIN AD, HIGGINS SA, URVA $S$, RATNER E et al. Phase I clinical trial of the mammalian target of rapamycin inhibitor everolimus in combination with oral topotecan for recurrent and advanced endometrial cancer. Int J Gynecol Cancer 2014; 24: 528-533. http://dx.doi. org/10.1097/IGC.0000000000000085

[16] ZHU M, GUO J, LI W, LU Y, FU S et al. Hepatitis B virus X protein induces expression of alpha-fetoprotein and activates $\mathrm{PI} 3 \mathrm{~K} / \mathrm{mTOR}$ signaling pathway in liver cells. Oncotarget 2015; 6: 12196-12208. http://dx.doi.org/10.18632/oncotarget.2906

[17] ASHWORTH RE, WU J. Mammalian target of rapamycin inhibition in hepatocellular carcinoma. World J Hepatol 2014; 6: 776-782. http://dx.doi.org/10.4254/wjh.v6.i11.776

[18] BEUVINK I, BOULAY A, FUMAGALLI S, ZILBERMANN F, RUETZ $S$ et al. The mTOR inhibitor RAD001 sensitizes tumor cells to DNA-damaged induced apoptosis through inhibition of p21 translation. Cell 2005; 120: 747-759. http:// dx.doi.org/10.1016/j.cell.2004.12.040

[19] GROTH CG, BACKMAN L, MORALES JM, CALNE R, KREIS $\mathrm{H}$ et al. Sirolimus (rapamycin)-based therapy in human renal transplantation: similar efficacy and different toxicity compared with cyclosporine. Sirolimus European Renal Transplant Study Group. Transplantation 1999; 67: 1036-1042. http:// dx.doi.org/10.1097/00007890-199904150-00017

[20] BRATTSTROM C, TYDEN G, SAWE J, HERLENIUS $\mathrm{G}, \mathrm{CLAESSON} \mathrm{K}$ et al. A randomized, double-blind, 
placebo-controlled study to determine safety, tolerance, and preliminary pharmacokinetics of ascending single doses of orally administered sirolimus (rapamycin) in stable renal transplant recipients. Transplant Proc 1996; 28: 985-986.

[21] Drug label of Oxaliplatin for Injection. Sanofi-Aventis France. Paris, France.

[22] KLINTMALM GB, NASHAN B. The role of mTOR inhibitors in liver transplantation: Reviewing the evidence. J Transplant 2014; 2014: 845438.

[23] RAHIMI RS, TROTTER JF. Liver transplantation for hepatocellular carcinoma: outcomes and treatment options for recurrence. Ann Gastroenterol. 2015; 28: 323-330.
[24] DEGLI ESPOSTI M, DIVE C. Mitochondrial membrane permeabilisation by Bax/Bak. Biochem Biophys Res Commun 2003; 304: 455-461. http://dx.doi.org/10.1016/S0006-291$\underline{\mathrm{X}(03) 00617-\mathrm{X}}$

[25] ROBBINS D, ZHAO Y. Oxidative stress induced by MnSODp53 interaction: Pro- or anti-tumorigenic? J Signal Transduct 2012; 2012: 101465.

[26] CHIPUK JE, KUWANA T, BOUCHIER-HAYES L, DROIN NM, NEWMEYER DD et al. Direct activation of Bax by p53 mediates mitochondrial membrane permeabilization and apoptosis. Science 2004; 303: 1010-1014. http://dx.doi. org/10.1126/science.1092734 
A: HepG2 cells


$\beta$-actin

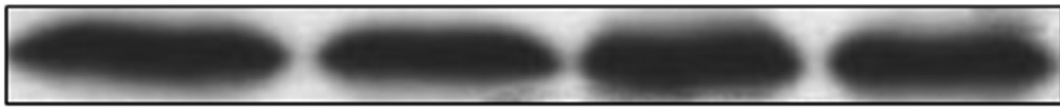

\section{B: BEL7402 cells}
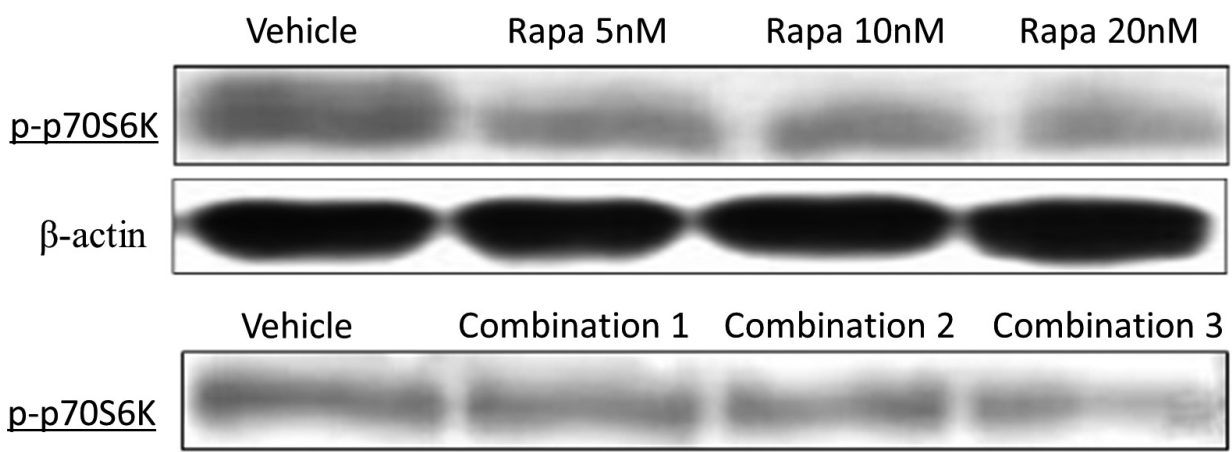

$\beta$-actin

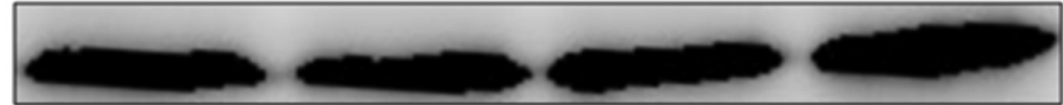

\section{C: HuH7 cells}

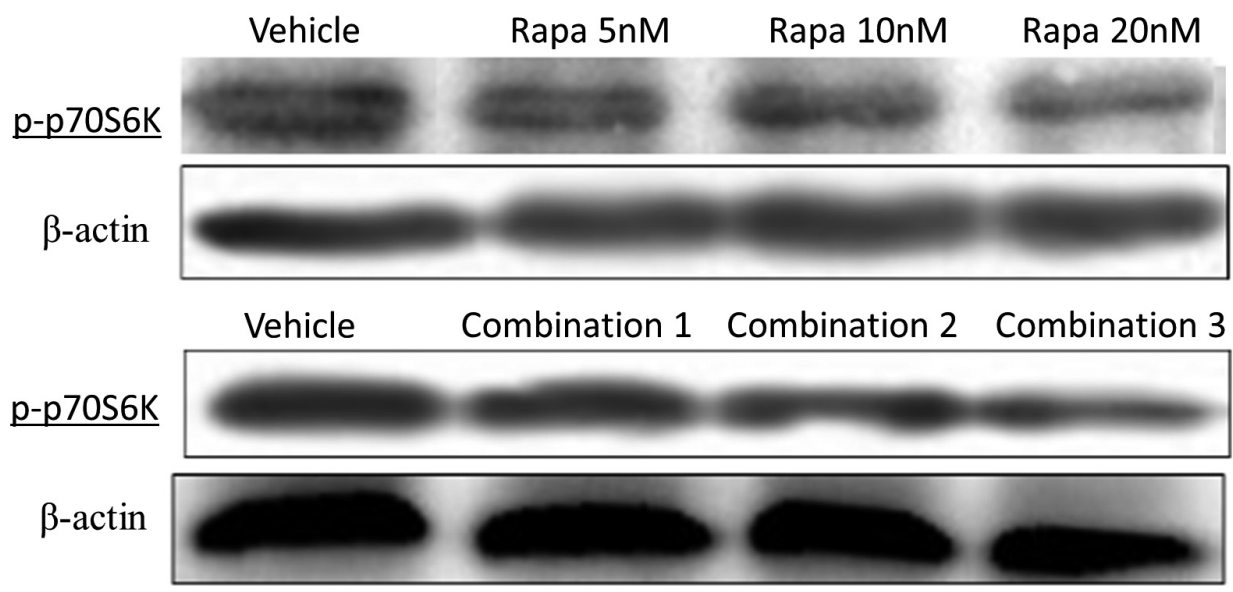

Supplementary Figure 1. The level of p-p70S6K in HepG2 (A), BEL7402 (B), and HuH7 (C) cells treated with rapamycin or rapamycin plus oxaliplatin. Cells were treated with vehicle, rapamycin alone or rapamycin plus oxaliplatin for $72 \mathrm{~h}$ and then subjected to Western blotting assay. Combination 1 , $5 \mathrm{nM}$ rapamycin $+2.5 \mu \mathrm{M}$ oxaliplatin; Combination $2,10 \mathrm{nM}$ rapamycin $+5 \mu \mathrm{M}$ oxaliplatin; Combination $3,20 \mathrm{nM}$ rapamycin $+10 \mu \mathrm{M}$ oxaliplatin. 
A HepG2
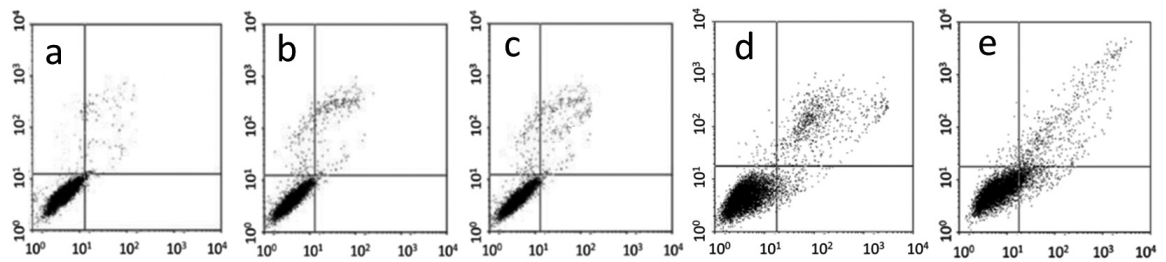

PI


Annexin V-FITC

B BEL7402

$\mathrm{PI}$
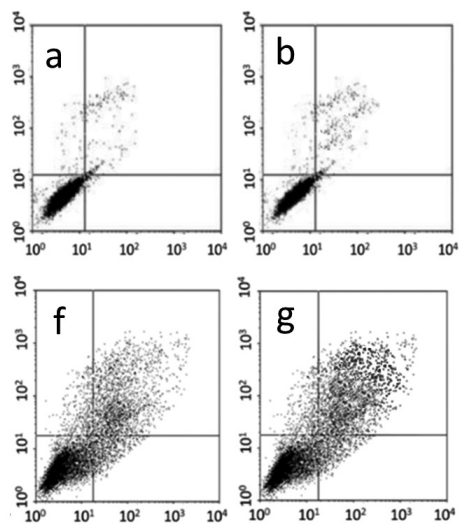
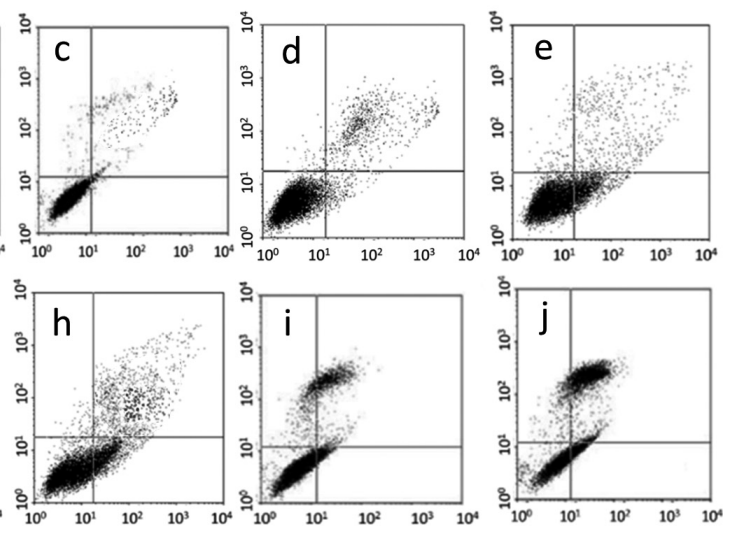

Annexin V-FITC

C $\mathrm{HuH7}$


Annexin V-FITC

Supplementary Figure 2. Original schematic on rapamycin, oxaliplatin, and their combination in soliciting HepG2 (A), BEL7402 (B), and HuH7 (C) cells apoptosis. Cells were treated with vehicle, rapamycin alone $(5,10$, and $20 \mathrm{nM}$, respectively), oxaliplatin alone $(2.5,5$, and $10 \mu \mathrm{M}$, respectively), and their combinations for $48 \mathrm{~h}$ and then subjected to flow cytometry analysis. a, vehicle; b, $5 \mathrm{nM}$ rapamycin; c, $10 \mathrm{nM}$ rapamycin; d, $20 \mathrm{nM}$ rapamycin; e, $2.5 \mu \mathrm{M}$ oxaliplatin; f, $5 \mu \mathrm{M}$ oxaliplatin; g, $10 \mu \mathrm{M}$ oxaliplatin; h, $5 \mathrm{nM}$ rapamycin plus $2.5 \mu \mathrm{M}$ oxaliplatin; i, $10 \mathrm{nM}$ rapamycin plus $5 \mu \mathrm{M}$ oxaliplatin; j, $20 \mathrm{nM}$ rapamycin plus $10 \mu \mathrm{M}$ oxaliplatin. 